TRANSACTIONS OF THE

AMERICAN MATHEMATICAL SOCIETY

Volume 351, Number 11, Pages 4561-4584

S 0002-9947(99)02509-X

Article electronically published on July 21, 1999

\title{
THE SUPPORT OF THE EQUILIBRIUM MEASURE IN THE PRESENCE OF A MONOMIAL EXTERNAL FIELD ON $[-1,1]$
}

\author{
S.B. DAMELIN AND A.B.J. KUIJLAARS
}

\begin{abstract}
The support of the equilibrium measure associated with an external field of the form $Q(x)=-c x^{2 m+1}, x \in[-1,1]$, with $c>0$ and $m$ a positive integer is investigated. It is shown that the support consists of at most two intervals. This resolves a question of Deift, Kriecherbauer and McLaughlin.
\end{abstract}

\section{INTRODUCTION AND STATEMENT OF MAIN RESULT}

The equilibrium measure in the presence of a continuous external field $Q$ : $[-1,1] \longrightarrow \mathbf{R}$ is the unique Borel probability measure $\mu$ on $[-1,1]$ satisfying, for some constant $F$,

$$
\begin{cases}U^{\mu}(x)+Q(x)=F, & x \in \operatorname{supp}(\mu) \\ U^{\mu}(x)+Q(x) \geq F, & x \in[-1,1] .\end{cases}
$$

Here $U^{\mu}$ denotes the logarithmic potential of $\mu$, i.e.,

$$
U^{\mu}(x):=\int_{-1}^{1} \log \frac{1}{|x-t|} d \mu(t) .
$$

We set

$$
w(x):=\exp (-Q(x)), \quad x \in[-1,1],
$$

and call $w$ the weight with respect to the external field $Q$. In the theory of weighted polynomials of the form $w^{n} P_{n}, n \geq 1$, a crucial role is played by the set $\operatorname{supp}(\mu)$. Indeed, Mhaskar and Saff [16] showed that for every polynomial $P_{n}$ of degree at most $n$,

$$
\left\|w^{n} P_{n}\right\|_{L_{\infty}([-1,1])}=\left\|w^{n} P_{n}\right\|_{L_{\infty}(\operatorname{supp}(\mu))},
$$

so that $w^{n} P_{n}$ actually "lives" on the set $\operatorname{supp}(\mu)$. Thus the determination of the equilibrium measure and its support is an important tool in describing asymptotics of weighted polynomials on $[-1,1]$.

Received by the editors June 22, 1997.

1991 Mathematics Subject Classification. Primary 31A15; Secondary 41A10, 45E05.

Key words and phrases. Balayage, equilibrium measure, external field, potential theory, weighted polynomials.

The research of the first author was begun while visiting the Mathematics Department at the Katholieke Universiteit Leuven, whose invitation to visit and hospitality are kindly acknowledged. This research was partly financed by FWO research project G.0278.97.

The second author was supported by a postdoctoral fellowship of the Belgian National Fund for Scientific Research, Scientific Research Network nr WO.011.96N: Fundamental Methods and Techniques in Mathematics. 
We also note that equilibrium problems like (1.1) play an important role in random matrix theory and the theory of singular limits of integrable systems. We refer the interested reader to [4], [3] and the references cited therein.

For convex external fields on $[-1,1]$, it is well known that the support of the equilibrium measure is an interval. As a result, weighted polynomials $w^{n} P_{n}$ with $Q(x)=-\log w(x)$ convex are studied frequently; see e.g. [7], [14], [17], [20] and also the recent books [13], [15], [18]. The corresponding theory for non-convex external fields, however, is less studied in the literature, and it is our purpose in this paper to investigate this subject further, building on the work of [11] and answering a question raised in [3].

More precisely, in this paper we study external fields of the form

$$
Q(x)=Q_{c}(x)=-c x^{2 m+1}, \quad x \in[-1,1],
$$

where $m$ is a fixed positive integer and $c>0$. The equilibrium measure in the presence of the external field $Q_{c}$ is denoted by $\mu_{c}$ and its support by $S_{c}$. Here the dependence on $m$ is not indicated explicitly. It follows that (1.1) takes the form

$$
\begin{cases}U^{\mu_{c}}(x)+Q_{c}(x)=F_{c}, & x \in S_{c} \\ U^{\mu_{c}}(x)+Q_{c}(x) \geq F_{c}, & x \in[-1,1],\end{cases}
$$

for some constants $F_{c}$, and that the above relations uniquely determine $\mu_{c}$.

Following is our main result.

Theorem 1.1. Let $m \in \mathbf{N}, m \geq 1$. Then there are three critical values $c_{j}=c_{j}(m)$, $j=1,2,3,0<c_{1}<c_{2}<c_{3}$, such that

(a) For $0<c \leq c_{1}$, we have

$$
S_{c}=[-1,1]
$$

(b) For $c_{1}<c \leq c_{2}$, we have

$$
S_{c}=[a, 1]
$$

with $a=a(c) \in(-1,0)$.

(c) For $c_{2}<c<c_{3}$, we have

$$
S_{c}=\left[a_{1}, b_{1}\right] \cup\left[a_{2}, 1\right]
$$

with $a_{j}=a_{j}(c) \in(-1,1), j=1,2, b_{1}=b_{1}(c)$ and $-1<a_{1}<b_{1}<a_{2}<1$.

(d) For $c_{3} \leq c<\infty$, we have

$$
S_{c}=[a, 1]
$$

with $a=a(c) \in(0,1)$.

Remark 1.2. Theorem 1.1 answers a question raised by Deift, Kriecherbauer and McLaughlin [3]. In that very interesting paper many new properties are derived for the equilibrium measure in the presence of an external field. For example, it is shown that for real analytic external fields, the support consists of a finite number of intervals. Furthermore, if the external field is a polynomial of degree $N$, then the number of intervals is at most $N+1$. As special cases they consider in detail the monomial external fields $-c x^{N}$, and they obtain our Theorem 1.1 for $N=3$. For odd $N \geq 5$, the part (c) was left open. For even $N$, see Remark 1.4 (2) below. 
Remark 1.3. We briefly mention the connection between fast decreasing polynomials on $[-1,1]$ and our problem. For a given external field $Q$ and $x_{0} \in[-1,1]$, we ask for a sequence of polynomials $\left\{P_{n}\right\}$ with $\operatorname{deg} P_{n} \leq n$, such that

$$
P_{n}\left(x_{0}\right)=e^{n Q\left(x_{0}\right)}
$$

and

$$
\left|P_{n}(x)\right| \leq e^{n(Q(x)+o(1))}, \quad x \in[-1,1] .
$$

For $x_{0}=0$ and $Q(x)=-c|x|^{\alpha}$ with fixed positive constants $c$ and $\alpha$, the polynomials $P_{n}$ satisfying (1.5) and (1.6) take the value 1 at the origin, while they decrease exponentially fast outside the origin, with a rate determined by the external field. This situation, in particular, was considered in [9], [12] and [21]. For further interesting applications of fast decreasing polynomials to weighted approximation on $[-1,1]$ and the real line, see [2], [5] and the references cited therein.

The connection between fast decreasing polynomials and potentials was thoroughly investigated by Totik in [21]; see also [18] and [20]. One of the main results in [21] is that, if $\mu$ is the equilibrium measure with the external field $Q$ as in (1.1), then fast decreasing polynomials satisfying (1.5) and (1.6) exist if and only if equality holds in (1.1) for $x=x_{0}$. In particular, a sufficient condition is that $x_{0} \in \operatorname{supp}(\mu)$.

If the external field is of the form $Q(x)=c Q_{1}(x), c>0$, then one may ask for the largest value of the parameter $c$ such that polynomials satisfying (1.5) and (1.6) exist. The answer is then given by

$$
\sup \left\{c>0: 0 \in S_{c}\right\}
$$

cf. equation (2.5) below.

Finallly, we note that for many external fields (including the fields given by (1.3)), the condition (1.6) can be replaced with the condition that

$$
\left|P_{n}(x)\right| \leq M e^{n Q(x)}, \quad x \in[-1,1],
$$

for some constant $M$, independent of $n$, see [18].

Remark 1.4. (1) In [11], Kuijlaars and Dragnev considered external fields of the form

$$
Q(x):=-c x^{\alpha}, \quad x \in[0,1], \quad \alpha>1,
$$

and they proved that for $\alpha>1$, the support consists of at most two intervals. To be precise, there are two critical values $c_{1}, c_{2}$, depending on $\alpha$. For $c \leq c_{1}$, the support is equal to the full interval $[0,1]$, for $c \in\left(c_{1}, c_{2}\right)$, the support has the form $[0, b] \cup[a, 1]$ with $0<b<a<1$, and for $c \geq c_{2}$, the support is an interval of the form $[a, 1]$. These results have applications to existence theorems for fast decreasing polynomials on $[0,1]$, see [12], [21].

Note that for $\alpha \leq 1$, the external field (1.5) is convex on $[0,1]$, so that in that case the support is always a single interval.

(2) The corresponding problem for external fields

$$
Q(x):=-c|x|^{\beta}, \quad x \in[-1,1], \quad \beta>2,
$$

can be resolved completely using the above mentioned result from [11] by a suitable quadratic transformation. The result is that there are two critical values $c_{1}, c_{2}$ which depend on $\beta$. For $c \leq c_{1}$, the support is $[-1,1]$, for $c \in\left(c_{1}, c_{2}\right)$ the support 
consists of three intervals and has the form $[-1,-a] \cup[-b, b] \cup[a, 1]$ with $0<b<$ $a<1$, and for $c \geq c_{2}$, the support consists of two intervals $[-1,-a] \cup[a, 1]$.

We note that this problem with $\beta=2 m$ an even integer was also considered in [3]. The cases $m=1$ and $m=2$ were covered completely, but for $m \geq 3$, there was also a gap in the range of parameters.

(3) What remains open is the investigation of the odd external fields

$$
Q(x):=-c \operatorname{sgn}(x)|x|^{\alpha}, \quad x \in[-1,1],
$$

when $\alpha$ is not an odd integer. We intend to come back to this in a future paper.

The rest of the paper is organized as follows. In Section 2 we collect together some general facts concerning the supports $S_{c}$ which will be needed in the proof of Theorem 1.1. In Section 3 we discuss balayage onto a finite number of intervals. We prove a result on the monotonic behavior of the density of a balayage measure. In Section 4 we study the iterated balayage algorithm of P. Dragnev, which was also used in [11]. This iterative method to solve the equilibrium problem (1.1) gives rise to a sequence of signed measures on $[-1,1]$, which in certain cases converges to the equilibrium measure $\mu$. We analyse this algorithm for polynomial external fields $Q$ and we show that convergence to $\mu$ takes place. As an aside, we recover the result from [3] that the support of the equilibrium measure consists of at most $\operatorname{deg} Q+1$ intervals. Combining the convergence of the iterated balayage algorithm with the result of Section 3, we arrive at Theorem 4.3 which extends a previous result of [11]. Finally, in Section 5, we prove our main result, Theorem 1.1.

Acknowledgments. The authors wish to thank P. Deift, T. Kriecherbauer and K. T.-R. McLaughlin for raising their interest in the above problem and for providing them with a copy of [3] prior to publication. We thank Doron Lubinsky, Vilmos Totik and Walter Van Assche for helpful discussions and encouragement.

\section{Preliminaries}

We now collect together some facts concerning the supports $S_{c}$ and their closely related "cousins"

$$
S_{c}^{*}:=\left\{x \in[-1,1]: U^{\mu_{c}}(x)+Q_{c}(x)=F_{c}\right\} .
$$

These facts are not specific to the external fields (1.3), but they apply to any family of external fields $Q_{c}=c Q_{1}, c>0$, with $Q_{1}$ a continuous function on [-1,1]. Most of these results can be found in the monograph of Saff and Totik [18].

Remark 2.1. (1) Note first that by (1.4) and (2.1) we have

$$
S_{c} \subset S_{c}^{*}, \quad c>0 .
$$

In general, equality need not hold.

(2) Next, observe that $Q_{c}$ has its minimum at $x=1$, and therefore by (1.2)

$$
1 \in S_{c}, \quad c>0 .
$$

In the general case of a family $Q_{c}=c Q_{1}$ of continuous external fields, this is

$$
\left\{x_{0} \in[-1,1]: Q_{1}\left(x_{0}\right)=\min _{x \in[-1,1]} Q_{1}(x)\right\} \subset S_{c}, \quad c>0 .
$$

(3) The support $S_{c}$ is decreasing as $c$ increases, i.e.,

$$
c<d \Longrightarrow S_{c} \supset S_{d}
$$


and $c \mapsto S_{c}$ is continuous from the right with respect to the Hausdorff metric on compact subsets of $[-1,1]$. This means in view of $(2.3)$ that

$$
S_{c}=\overline{\bigcup_{d>c} S_{d}}
$$

For this, see Theorems IV.1.6 and IV.4.1 of [18].

(4) The mapping $c \mapsto S_{c}$ is not necessarily continuous from the left. That is, we have by (2.3) that

$$
S_{c} \subset \bigcap_{d<c} S_{d}, \quad c>0,
$$

but the inclusion may be strict. Instead, we always have

$$
S_{c}^{*}=\bigcap_{d<c} S_{d}, \quad c>0,
$$

where $S_{c}^{*}$ is given by (2.1). It follows that $c \mapsto S_{c}$ is continuous at a point $c$ if and only if $S_{c}=S_{c}^{*}$; see Theorem IV.1.6 (g) of [18].

We note that it will follow from Theorem 1.1 that $S_{c}=S_{c}^{*}$ for all $c>0$, except $c=c_{3}$.

The relation (2.5) can be deduced easily from results in [18], in particular Theorem IV.4.1 and Lemma IV.4.3. We thank V. Totik for this remark. We will give here a simple proof based on the following lemma.

Lemma 2.2. Let $Q$ be a continuous external field on $[-1,1]$ with equilibrium measure $\mu$ characterized by the equations (1.1). Let $\nu \neq \mu$ be a non-negative Borel measure on $\mathbf{C}$ with a compact support and $\|\nu\| \leq 1$. If $x_{0} \in \mathbf{C}$ is such that

$$
U^{\mu-\nu}\left(x_{0}\right) \geq \max _{x \in \operatorname{supp}(\mu)} U^{\mu-\nu}(x),
$$

then $x_{0} \in \operatorname{supp}(\mu)$.

Proof. The function $U^{\mu-\nu}$ is subharmonic outside the support of $\mu$, and also at $\infty$, since $\|\nu\| \leq 1=\|\mu\|$. Therefore, by the maximum principle, the maximum of $U^{\mu-\nu}$ is attained in $\operatorname{supp}(\mu)$. Moreover, if the maximum is also attained at a point outside of $\operatorname{supp}(\mu)$, then $U^{\mu-\nu}$ is necessarily a constant throughout $\mathbf{C}$, and this is impossible, because $\mu \neq \nu$. Thus every $x_{0}$ satisfying (2.6) belongs to $\operatorname{supp}(\mu)$.

Proof of (2.5). The fact that $\bigcap_{d<c} S_{d} \subset S_{c}^{*}$ is contained in Theorem IV.4.1 of [18]. It is therefore enough to prove that $S_{c}^{*} \subset S_{d}$ whenever $d<c$.

Thus let $d<c$ and put $Q=Q_{d}, \mu=\mu_{d}$ and $\nu=(d / c) \mu_{c}$. For $x \in \operatorname{supp}(\mu)=S_{d}$, we have, by (1.4),

$$
\begin{aligned}
U^{\mu-\nu}(x) & =\left(U^{\mu}(x)+Q(x)\right)-\left(U^{\nu}(x)+Q(x)\right) \\
& =\left(U^{\mu_{d}}(x)+Q_{d}(x)\right)-(d / c)\left(U^{\mu_{c}}(x)+Q_{c}(x)\right) \\
& \leq F_{d}-(d / c) F_{c} .
\end{aligned}
$$

Thus

$$
\max _{x \in \operatorname{supp}(\mu)} U^{\mu-\nu}(x) \leq F_{d}-(d / c) F_{c} .
$$


Next, if $x_{0} \in S_{c}^{*}$, then, by (1.4) and (2.1),

$$
\begin{aligned}
U^{\mu-\nu}\left(x_{0}\right) & =\left(\left(U^{\mu_{d}}\left(x_{0}\right)+Q_{d}\left(x_{0}\right)\right)-(d / c)\left(U^{\mu_{c}}\left(x_{0}\right)+Q_{c}\left(x_{0}\right)\right)\right. \\
& \geq F_{d}-(d / c) F_{c} \\
& \geq \max _{x \in \operatorname{supp}(\mu)} U^{\mu-\nu}(x) .
\end{aligned}
$$

This implies $x_{0} \in S_{d}$ by Lemma 2.2 , since $\|\nu\| \leq 1$ and $\nu \neq \mu$.

We finally need an extension of (2.4) that characterizes the set $\bigcup_{d>c} S_{d}$ (without closure). We will state this result for a family of external fields $Q_{c}=c Q_{1}$ that are real analytic in a neighborhood of $[-1,1]$. For a field $Q$ which is real analytic in a neighborhood of $[-1,1]$, it was shown in [3] that the equilibrium measure $\mu$ is supported on a finite number of intervals. Furthermore, there is a function $h$, real analytic in a neigborhood of $[-1,1]$, such that

$$
\operatorname{supp}(\mu)=\overline{\{t \in[-1,1]: h(t)>0\}},
$$

and if we write $h=h^{+}-h^{-}$, where $h^{+}$and $h^{-}$are the positive and negative parts of $h$, respectively, then

$$
\frac{d \mu(t)}{d t}=\frac{\sqrt{h^{+}(t)}}{\pi \sqrt{1-t^{2}}}, \quad t \in[-1,1] .
$$

For this, see Theorems 1.34 and 1.38 of [3].

Lemma 2.3. Let $Q_{c}=c Q_{1}$ be a family of external fields that are real analytic in a neighborhood of $[-1,1]$. Let $\mu_{c}$ be the equilibrium measure with external field $Q_{c}$ on $[-1,1]$, and let $S_{c}=\operatorname{supp}\left(\mu_{c}\right)$. Then

$$
\bigcup_{d>c} S_{d}=\left\{x_{0} \in S_{c}: \frac{d \mu_{c}}{d t}\left(x_{0}\right)>0\right\} .
$$

Proof. In the proof we use $v_{c}$ to denote the density of $\mu_{c}$.

Let $d>c$. We use Lemma 5.7 of [20], which says that

$$
\left(\mu_{c}\right)_{\left.\right|_{S_{d}}} \geq \frac{c}{d} \mu_{d}+(1-c / d)\left(\omega_{S_{c}}\right)_{S_{d}},
$$

where $\omega_{S_{c}}$ is the equilibrium measure (without external field) of $S_{c}$. In terms of densities, this is

$$
v_{c}\left(x_{0}\right) \geq \frac{c}{d} v_{d}\left(x_{0}\right)+(1-c / d) \frac{d \omega_{S_{c}}}{d t}\left(x_{0}\right), \quad x_{0} \in S_{d} .
$$

The equilibrium measure $\omega_{S_{c}}$ has a density that does not vanish on $S_{c}$. So we get, since $S_{d} \subset S_{c}$,

$$
v_{c}\left(x_{0}\right) \geq(1-c / d) \frac{d \omega_{S_{c}}}{d t}\left(x_{0}\right)>0, \quad x_{0} \in S_{d} .
$$

We have shown that $\bigcup_{d>c} S_{d}$ is contained in $\left\{x_{0} \in S_{c}: v_{c}\left(x_{0}\right)>0\right\}$.

For the converse, we suppose that $v_{c}\left(x_{0}\right)>0$. The proof that $x_{0} \in S_{d}$ for some $d>c$ is based on the fact that there exists a non-negative measure $\rho$ on $S_{c}$ satisfying

$$
\rho \leq \mu_{c}, \quad \rho \neq 0,
$$

and

$$
U^{\rho}\left(x_{0}\right)=\max _{x} U^{\rho}(x)
$$


Before we prove (2.10), (2.11) let us first show how to obtain from it the result that $x_{0} \in S_{d}$ for some $d>c$.

Assuming (2.10), (2.11), we write $\delta:=\int d \rho$, so that $0<\delta \leq 1$ by (2.10). We pick $d$ such that

$$
c<d<\frac{c}{1-\delta}
$$

and we define the measure

$$
\nu:=\frac{d}{c}\left(\mu_{c}-\rho\right) .
$$

Then $\nu$ is a non-negative measure with $\|\nu\|<1$. For $x \in S_{d}$, we have

$$
\begin{aligned}
U^{\mu_{d}-\nu}(x) & =U^{\mu_{d}}(x)+Q_{d}(x)-\frac{d}{c}\left(U^{\mu_{c}}(x)+Q_{c}(x)\right)+\frac{d}{c} U^{\rho}(x) \\
& \leq F_{d}-\frac{d}{c} F_{c}+\frac{d}{c} U^{\rho}\left(x_{0}\right) .
\end{aligned}
$$

Here we used (1.4) and (2.11). For $x_{0}$, we get

$$
\begin{aligned}
U^{\mu_{d}-\nu}\left(x_{0}\right) & =U^{\mu_{d}}\left(x_{0}\right)+Q_{d}\left(x_{0}\right)-\frac{d}{c}\left(U^{\mu_{c}}\left(x_{0}\right)+Q_{c}\left(x_{0}\right)\right)+\frac{d}{c} U^{\rho}\left(x_{0}\right) \\
& \geq F_{d}-\frac{d}{c} F_{c}+\frac{d}{c} U^{\rho}\left(x_{0}\right),
\end{aligned}
$$

because of (1.4) and the fact that $x_{0} \in S_{c}$. Thus from (2.12), (2.13), we have

$$
U^{\mu_{d}-\nu}\left(x_{0}\right) \geq \max _{x \in S_{d}} U^{\mu_{d}-\nu}(x)
$$

and this implies $x_{0} \in S_{d}$ by Lemma 2.2 , since $\|\nu\|<1$.

So what remains is to prove the existence of a measure $\rho$ satisfying $(2.10),(2.11)$. We note that by (2.7) and (2.8), there is a real analytic function $h_{c}$ on a neighborhood of $[-1,1]$ such that

$$
v_{c}(t)=\frac{\sqrt{h_{c}^{+}(t)}}{\pi \sqrt{1-t^{2}}}, \quad t \in[-1,1] .
$$

If $x_{0}$ is an interior point of $S_{c}$, then, since $v_{c}$ is continuous by (2.14), there are $\epsilon_{0}, \epsilon_{1}>0$ such that $v_{c}(t) \geq \epsilon_{0}$ on $\left[x_{0}-\epsilon_{1}, x_{0}+\epsilon_{1}\right]$. Let $\rho$ be the measure with density $\epsilon_{0}$ on $\left[x_{0}-\epsilon_{1}, x_{0}+\epsilon_{1}\right]$ and 0 elsewhere. Then (2.10) is clearly satisfied, and (2.11) is easily verified by direct calculation.

If $x_{0}$ is a boundary point of $S_{c}$ and $v_{c}\left(x_{0}\right)>0$, then it follows from (2.14) that we have either $x_{0}=-1$ or $x_{0}=1$. Suppose without loss of generality that $x_{0}=1$. Then there exist $\epsilon_{0}, \epsilon_{1}>0$ such that

$$
v_{c}(t) \geq \epsilon_{0}|1-t|^{-1 / 2}, \quad t \in\left[1-\epsilon_{1}, 1\right] .
$$

This follows from (2.14) and the fact that $h_{c}$ is real analytic in a neighborhood of $[-1,1]$. Let $\rho$ be the measure with density $\epsilon_{0}|1-t|^{-1 / 2}$ for $t \in\left[1-\epsilon_{1}, 1\right]$, and 0 elsewhere. Then again it is easily shown that $\rho$ satisfies (2.10) and (2.11).

This completes the proof of the lemma.

Remark 2.4. Lemma 2.3 should be compared with Lemma 5.8 of [20]. If $v_{c}\left(x_{0}\right)>0$ and $x_{0}$ is an interior point of $S_{c}$, then this lemma immediately gives that $x_{0} \in S_{d}$ for some $d>c$, which is, of course, part of the statement (2.9). We have chosen to include a full proof also for this case, since it is simpler than the proof in [20] and 
the same proof works for boundary point $x_{0}$, which is not covered by the results of [20].

\section{BAlayage onto A Finite NUMBer of Intervals}

We recall the notion of balayage onto a compact set, see [18] or [19]. Let $K$ be a compact subset of the complex plane with positive logarithmic capacity and such that the complement $\overline{\mathbf{C}} \backslash K$ is regular for the Dirichlet problem. Then, if $\nu$ is any finite positive Borel measure on $\mathbf{C}$ with compact support, there exists a unique measure $\hat{\nu}$ supported on $K$ such that $\|\nu\|=\|\hat{\nu}\|$, and for some constant $C$,

$$
U^{\hat{\nu}}(z)=U^{\nu}(z)+C, \quad z \in K .
$$

The measure $\hat{\nu}$ is called the balayage of $\nu$ onto $K$ and we denote it by $\operatorname{Bal}(\nu ; K)$. For a signed measure $\sigma=\sigma^{+}-\sigma^{-}$, we define $\operatorname{Bal}(\sigma ; K):=\operatorname{Bal}\left(\sigma^{+} ; K\right)-\operatorname{Bal}\left(\sigma^{-} ; K\right)$.

We are going to take the balayage of measures onto sets $\Sigma$ which are finite unions of closed disjoint intervals on the real line. The main result of this section is the following:

Theorem 3.1. Let $\Sigma=\bigcup_{j=1}^{l}\left[a_{j}, b_{j}\right]$ be a finite union of closed disjoint intervals with

$$
a_{1}<b_{1}<a_{2}<\cdots<a_{l}<b_{l} .
$$

Let $\nu$ be a finite positive Borel measure supported on the union of the gaps, i.e., on $\bigcup_{j=1}^{l-1}\left(b_{j}, a_{j+1}\right)$. Then the measure Bal $(\nu ; \Sigma)$ has a density $v$ on $\Sigma$ such that

$$
\sqrt{\left(t-a_{1}\right)\left(b_{l}-t\right)} v(t), \quad t \in \Sigma,
$$

increases on the first interval $\left[a_{1}, b_{1}\right]$ and decreases on the last interval $\left[a_{l}, b_{l}\right]$.

We need the following lemma.

Lemma 3.2. Let $\Sigma=\bigcup_{j=1}^{l}\left[a_{j}, b_{j}\right]$ with $a_{1}<b_{1}<a_{2}<\cdots<a_{l}<b_{l}$ be as in Theorem 3.1. Fix a point $s \in\left(b_{j^{*}}, a_{j^{*}+1}\right)$ for some $j^{*} \leq l-1$, and let $\delta_{s}$ be the point mass at $s$. Then

$$
\frac{d\left(\operatorname{Bal}\left(\delta_{s} ; \Sigma\right)\right)}{d t}=\frac{T_{s}(t)}{(t-s) R(t)}, \quad t \in \Sigma
$$

where

$$
R(t):=(-1)^{l-j_{0}}\left(\prod_{j=1}^{l}\left|\left(b_{j}-t\right)\left(t-a_{j}\right)\right|\right)^{1 / 2}, \quad t \in\left(a_{j_{0}}, b_{j_{0}}\right), \quad j_{0}=1, \ldots, l,
$$

and $T_{s}$ is a polynomial of degree $\leq l-1$ with exactly one zero in each of the gaps $\left(b_{j}, a_{j+1}\right), j \neq j^{*}$. In addition, there is one more zero in $\mathbf{R} \backslash\left[a_{1}, b_{l}\right]$ if the degree of $T_{s}$ is $l-1$.

Remark 3.3. We have defined $R$ in (3.4) in such a way that its sign changes when going from one interval to the next. Because of the properties of the zeros of $T_{s}$, also $T_{s}(t) /(t-s)$ changes sign, and we see that the right hand side of (3.3) has constant (positive) sign.

Note that the polynomials $T_{s}$ depend on $\Sigma$ as well. 
Proof of Lemma 3.2. Without loss of generality, we may assume that $s=0$. We define

$$
\Sigma^{-1}:=\left\{x^{-1}: x \in \Sigma\right\} .
$$

Note that as $0 \notin \Sigma$, the set $\Sigma^{-1}$ is compact and it is the union of $l$ closed intervals. Let $\omega$ denote the usual equilibrium measure (without external field) for $\Sigma^{-1}$ and let $\rho$ be the image of the measure $\omega$ under the mapping $t \mapsto 1 / t$. We claim that

$$
\operatorname{Bal}\left(\delta_{0} ; \Sigma\right)=\rho .
$$

To see this, we note first that $\rho$ is a probability measure supported on $\Sigma$. By the uniqueness of balayage it then suffices to show that

$$
U^{\rho}(x)=U^{\delta_{0}}(x)+C
$$

for some constant $C$ and for every $x \in \Sigma$. We have, for $x \in \Sigma$,

$$
\begin{aligned}
U^{\rho}(x) & =\int_{\Sigma} \log \frac{1}{|x-t|} d \rho(t) \\
& =\int_{\Sigma^{-1}} \log \frac{1}{\left|x-t^{-1}\right|} d \omega(t) \\
& =\log \frac{1}{|x|}+\int_{\Sigma^{-1}} \log |t| d \omega(t)+\int_{\Sigma^{-1}} \log \frac{1}{\left|x^{-1}-t\right|} d \omega(t) \\
& =U^{\delta_{0}}(x)-U^{\omega}(0)+U^{\omega}\left(x^{-1}\right),
\end{aligned}
$$

with $x^{-1} \in \Sigma^{-1}$. Since $\omega$ is the equilibrium measure of $\Sigma^{-1}$, its potential is constant on $\Sigma^{-1}$ and this constant is equal to $-\log \left(\operatorname{cap}\left(\Sigma^{-1}\right)\right)$, where cap $\left(\Sigma^{-1}\right)$ is the logarithmic capacity of $\Sigma^{-1}$. We have proved (3.6) with $C=-U^{\omega}(0)-\log \left(\operatorname{cap}\left(\Sigma^{-1}\right)\right)$, and our claim (3.5) follows.

Now (3.4) follows from the known representation of the equilibrium measure $\omega$ of $\Sigma^{-1}$ (see for example Lemma 4.4.1 of [19]).

Proof of Theorem 3.1. By symmetry, it suffices to show that (3.2) increases on $\left[a_{1}, b_{1}\right]$.

From Lemma 3.2 it follows that the balayage of $\nu$ onto $\Sigma$ has the density

$$
v(t)=\int \frac{T_{s}(t)}{(t-s) R(t)} d \nu(s), \quad t \in \Sigma,
$$

where $T_{s}$ and $R$ are as in Lemma 3.2. Here we used the fact that

$$
\operatorname{Bal}(\nu ; \Sigma)=\int \operatorname{Bal}\left(\delta_{s} ; \Sigma\right) d \nu(s),
$$

see e.g. formula (4.13) of Chapter II in [18]. We see that by (3.7) it is enough to show that

$$
\sqrt{\left(t-a_{1}\right)\left(b_{l}-t\right)} \frac{T_{s}(t)}{(t-s) R(t)}
$$

increases on $\left[a_{1}, b_{1}\right]$ for every $s \in\left[a_{1}, b_{l}\right] \backslash \Sigma$.

We first consider the case that $s$ belongs to the gap $\left(b_{1}, a_{2}\right)$. Then by Lemma 3.2 we can write

$$
T_{s}(t)=C(1-t Y) \prod_{j=2}^{l-1}\left(t-y_{j}\right)
$$


with $y_{j} \in\left(b_{j}, a_{j+1}\right)$ for $j=2, \ldots, l-1, Y^{-1} \in \overline{\mathbf{R}} \backslash\left[a_{1}, b_{l}\right]$ and $C$ a non-zero constant (which all depend on $\Sigma$ and $s$ ). Then (3.8) is

$$
\frac{C|1-t Y|}{|t-s|} \frac{1}{\sqrt{b_{1}-t}} \prod_{j=2}^{l-1} \frac{y_{j}-t}{\sqrt{\left(a_{j}-t\right)\left(b_{j}-t\right)}} \frac{1}{\sqrt{a_{l}-t}}, \quad t \in\left(a_{1}, b_{1}\right) .
$$

It is easy to see that all factors in (3.9) are positive and increasing on $\left[a_{1}, b_{1}\right]$. Therefore, the product is increasing on $\left[a_{1}, b_{1}\right]$, and it follows that (3.8) increases on $\left[a_{1}, b_{1}\right]$ for every $s \in\left(b_{1}, a_{2}\right)$. Using (3.7) we see that we have proved that (3.2) increases on $\left[a_{1}, b_{1}\right]$ if $\nu$ is supported in the first gap $\left(b_{1}, a_{2}\right)$.

Using mathematical induction, we may assume that (3.2) increases on $\left[a_{1}, b_{1}\right]$ for all positive finite measures supported on the union of the first $j^{*}-1$ gaps with $j^{*} \geq 2$, and we may further assume that $\nu$ is supported on $\left(b_{j^{*}}, a_{j^{*}+1}\right)$. Put

$$
\Sigma_{1}=\left[a_{1}, b_{j^{*}}\right] \cup \Sigma,
$$

which is a finite union of intervals, and $\nu$ is supported on the first gap of $\Sigma_{1}$. Therefore, by what has been proved before, the balayage measure $\operatorname{Bal}\left(\nu ; \Sigma_{1}\right)$ onto $\Sigma_{1}$ has a density $v_{1}$, and

$$
\sqrt{\left(t-a_{1}\right)\left(b_{l}-t\right)} v_{1}(t)
$$

is increasing on $\left[a_{1}, b_{j^{*}}\right]$, and so in particular on $\left[a_{1}, b_{1}\right]$. Let $\nu_{1}$ be the measure $\operatorname{Bal}\left(\nu ; \Sigma_{1}\right)$ restricted to $\bigcup_{j=1}^{j^{*}-1}\left(b_{j}, a_{j+1}\right)$, and let $v_{2}$ be the density of the balayage measure $\operatorname{Bal}\left(\nu_{1} ; \Sigma\right)$. By hypothesis, we have that

$$
\sqrt{\left(t-a_{1}\right)\left(b_{l}-t\right)} v_{2}(t)
$$

increases on $\left[a_{1}, b_{1}\right]$. Since $v=v_{1}+v_{2}$ on $\Sigma$, we get that (3.2) increases on $\left[a_{1}, b_{1}\right]$ if $\nu$ is supported on $\left(b_{j^{*}}, a_{j^{*}+1}\right)$.

This completes the proof of Theorem 3.1.

\section{The iterated BALAYAge ALGORIthm}

The iterated balayage algorithm gives an iterative method for solving an equilibrium problem (1.1) with an external field.

Given the external field $Q$ on $[-1,1]$, one proceeds as follows. Suppose one knows that the support of $\mu$ is contained in the interval $[a, b]$. (For example, one could take $[-1,1]$, but it will be useful to have some freedom here.) Then the first step is to solve the integral equation

$$
\int_{a}^{b} \log |x-t| v_{0}(t) d t=Q(x)-F_{0}, \quad a<x<b,
$$

subject to the condition

$$
\int_{a}^{b} v_{0}(t) d t=1
$$

Formally differentiating (4.1) with respect to $x$, one obtains the singular integral equation

$$
f_{a}^{b} \frac{v_{0}(t)}{x-t} d t=Q^{\prime}(x), \quad a<x<b
$$

Here $f$ is used to denote a Cauchy principle value integral. 
It is well-known that if $Q$ is smooth enough, for example if $Q$ is differentiable with a Hölder continuous derivative, i.e., $Q \in C^{1+\epsilon}([a, b])$ for some $\epsilon>0$, then (4.2)-(4.3) has the unique solution

$$
v_{0}(t)=\frac{1}{\pi \sqrt{(b-t)(t-a)}}\left[1+\frac{1}{\pi} \int_{a}^{b} \frac{Q^{\prime}(s)}{s-t} \sqrt{(b-s)(s-a)} d s\right], \quad a<t<b,
$$

where the above integral is again a Cauchy principle value integral, see $\$ 42.3$ of [6]. If the function $v_{0}$ happens to be non-negative on $[a, b]$, then it is the density of the equilibrium measure with external field $Q$ and we are done. If not, then we put

$$
d \sigma_{0}(t):=v_{0}(t) d t
$$

so that $\sigma_{0}$ is a signed measure on $[a, b]$. Let $\sigma_{0}=\sigma_{0}^{+}-\sigma_{0}^{-}$be the Jordan decomposition of $\sigma_{0}$ and

$$
\Sigma_{1}:=\operatorname{supp}\left(\sigma_{0}^{+}\right) .
$$

It was shown in [11] that $\mu \leq \sigma_{0}^{+}$and $\operatorname{supp}(\mu) \subset \Sigma_{1}$, so that in determining $\mu$ and its support we may restrict ourselves to $\Sigma_{1}$. The next step is to consider the integral equation on $\Sigma_{1}$

$$
\int_{\Sigma_{1}} \log |x-t| d \sigma_{1}(t)=Q(x)-F_{1}, \quad x \in \Sigma_{1}
$$

subject to the condition

$$
\int_{\Sigma_{1}} d \sigma_{1}=1
$$

The solution to (4.5)-(4.6) can be expressed in terms of the measure $\sigma_{0}$ : it is simply the balayage of $\sigma_{0}$ onto $\Sigma_{1}$, see (3.1).

To describe this process, an operator $J$ was introduced in [11] on all finite signed measures $\sigma$ on $[-1,1]$ with $\int d \sigma=1$ and $\operatorname{cap}\left(\operatorname{supp}\left(\sigma^{+}\right)\right)>0$ as follows:

$$
J(\sigma):=\sigma^{+}-\operatorname{Bal}\left(\sigma^{-} ; \operatorname{supp}\left(\sigma^{+}\right)\right)=\operatorname{Bal}\left(\sigma ; \operatorname{supp}\left(\sigma^{+}\right)\right) .
$$

The operator $J$ sweeps the negative part of the measure $\sigma$ onto the support of the positive part, so that in particular $J(\sigma)^{+} \leq \sigma^{+}$.

Returning to (4.5)-(4.6), we see that $\sigma_{1}$ is given by $\sigma_{1}=J\left(\sigma_{0}\right)$. Continuing in this way, we write, for every $k \geq 1$,

$$
\Sigma_{k}:=\operatorname{supp}\left(\sigma_{k-1}^{+}\right), \quad \sigma_{k}:=J\left(\sigma_{k-1}\right)=J^{k}\left(\sigma_{0}\right) .
$$

The measures $\sigma_{k}$ are signed measures which have a Jordan decomposition $\sigma_{k}=$ $\sigma_{k}^{+}-\sigma_{k}^{-}$. It follows as in [11] that

$$
\sigma_{0}^{+} \geq \sigma_{1}^{+} \geq \cdots \geq \mu
$$

and

$$
[a, b] \supset \Sigma_{1} \supset \Sigma_{2} \supset \cdots \supset \operatorname{supp}(\mu) .
$$

Under quite general conditions one expects from (4.8) that the sequence $\left\{\sigma_{k}^{+}\right\}_{k=0}^{\infty}$ converges in the weak* sense to the equilibrium measure $\mu$, but this has not been proven yet. If it holds, then we say that the iterated balayage algorithm converges.

Besides presenting a possible algorithm for numerical calculations, the iterated balayage algorithm can also be used to prove rigorous results on the support of $\mu$ in certain situations. This was done in [11], where for the class of external fields 
given in (1.5), it was shown that $\Sigma_{k}$ consists of at most two intervals for every $k$, which led to the result that the support of $\mu$ also consists of at most two intervals; see Remark 1.4 (1).

The main difficulty in proving that the iterated balayage algorithm converges generally lies in the fact that one has to show that the negative parts $\sigma_{k}^{-}$tend to zero as $k$ tends to $\infty$. This can be shown, if one can control the limiting set $\Sigma^{*}$. Indeed, any possible limit measure of a subsequence of $\left\{\sigma_{k}^{-}\right\}$is supported on $\partial \Sigma^{*}$, the topological boundary of $\Sigma^{*}$ in $[a, b]$. (This holds provided that for every $k$, $\Sigma_{k+1}$ is contained in the interior of $\Sigma_{k}$ with respect to $[-1,1]$, which will be true in many cases.) If the capacity of $\partial \Sigma^{*}$ is zero, then $\partial \Sigma^{*}$ cannot support a non-zero measure with a finite potential. This forces the sequence $\left\{\sigma_{k}^{-}\right\}$to converge to 0 , which proves the convergence of the iterated balayage algorithm.

Using these ideas, we consider the case of a polynomial external field. As a byproduct of the analysis we find that for a polynomial external field of degree $m$ on an interval, the support of the equilibrium measures consists of at most $m+1$ intervals. This was shown earlier by Deift, Kriecherbauer and McLaughlin [3] using different methods. This bound is not the best possible, since for $m=1$, the external field is convex and the support is therefore an interval. The optimal number is $(m / 2)+1$ if $m$ is even, and $(m+1) / 2$ if $m$ is odd, as remarked on page 408 of [3].

Theorem 4.1. Let $Q$ be a polynomial of degree $m$ and let $\mu$ be the equilibrium measure in the presence of the external field $Q$ on $[-1,1]$. Suppose the support of $\mu$ is contained in the interval $[a, b]$ and let $v_{0}, \sigma_{k}$, and $\Sigma_{k}$ be as in (4.4) and (4.7). Define

$$
\Sigma^{*}:=\bigcap_{k=1}^{\infty} \Sigma_{k}
$$

Then the following hold.

(a) For each $k$, the set $\Sigma_{k}$ consists of at most $m+1$ intervals, say

$$
\Sigma_{k}=\bigcup_{j=1}^{l_{k}}\left[a_{j}^{(k)}, b_{j}^{(k)}\right]
$$

with $l_{k} \leq m+1$ and $a_{1}^{(k)}<b_{1}^{(k)}<\cdots<b_{l_{k}}^{(k)}$.

(b) For each $k$, the signed measure $\sigma_{k}$ has a density $v_{k}$ on $\Sigma_{k}$, and if

$$
R_{k}(t):=(-1)^{l_{k}-j_{0}}\left(\prod_{j=1}^{l_{k}}\left|\left(b_{j}^{(k)}-t\right)\left(t-a_{j}^{(k)}\right)\right|\right)^{1 / 2}, \quad t \in\left(a_{j_{0}}^{(k)}, b_{j_{0}}^{(k)}\right),
$$

then

$$
P_{k}(t):=\pi R_{k}(t) v_{k}(t), \quad t \in \Sigma_{k},
$$

is a polynomial of degree $m+l_{k}-1$. (More precisely, the restriction of a polynomial to $\Sigma_{k}$, but we will not bother about such distinctions here and in the sequel.)

(c) The measures $\sigma_{k}^{+}$converge to the measure $\mu$ in the weak ${ }^{*}$ sense.

(d) The support of $\mu$ is equal to $\Sigma^{*}$ minus its isolated points.

(e) The support of $\mu$ consists of at most $m+1$ intervals. 
First we need a lemma.

Lemma 4.2. Let $\Sigma=\bigcup_{j=1}^{l}\left[a_{j}, b_{j}\right]$ with $a_{1}<b_{1}<\cdots<b_{l}$, and let

$$
R(t)=(-1)^{l-j_{0}}\left(\prod_{j=1}^{l}\left|\left(b_{j}-t\right)\left(t-a_{j}\right)\right|\right)^{1 / 2}, \quad t \in\left(a_{j_{0}}, b_{j_{0}}\right) .
$$

Let $Q$ be a polynomial of degree $m$. Then

$$
P(t):=\int_{\Sigma} \frac{Q^{\prime}(s)}{s-t} R(s) d s
$$

is a polynomial of degree $m+l-1$ exactly.

Proof. Since $R^{2}$ is a polynomial of degree $2 l$, we see that $Q^{\prime} R^{2}$ has degree $m+2 l-1$. We can write $Q^{\prime} R^{2}=A B$, where $A$ and $B$ are polynomials of degrees $m+l$ and $l-1$, respectively. Then

$$
\begin{aligned}
P(t) & =\int_{\Sigma} \frac{A(s) B(s)}{s-t} \frac{d s}{R(s)} \\
& =\int_{\Sigma} \frac{A(s)-A(t)}{s-t} \frac{B(s) d s}{R(s)}+A(t) f_{\Sigma} \frac{B(s)}{s-t} \frac{d s}{R(s)} .
\end{aligned}
$$

In the final expression, the first integral is a polynomial of degree $m+l-1$, since for each $s,(A(s)-A(t)) /(s-t)$ is a polynomial of degree $m+l-1$ in $t$. The second integral vanishes identically, since the degree of $B$ is $l-1$, see $\S 42.3$ of [6].

Proof of Theorem 4.1. From Lemma 4.2 and (4.4) we get that

$$
P_{0}(t):=\pi \sqrt{(b-t)(t-a)} v_{0}(t), \quad a<t<b,
$$

is a polynomial of degree $m$. Thus $P_{0}$ has at most $m$ zeros in $[a, b]$, which implies that $\Sigma_{1}$ consists of at most $[m / 2]+1$ intervals, say

$$
\Sigma_{1}:=\bigcup_{j=1}^{l_{1}}\left[a_{j}^{(1)}, b_{j}^{(1)}\right]
$$

with $l_{1} \leq[m / 2]+1$ and $a_{1}^{(1)}<b_{1}^{(1)}<\cdots<b_{l_{1}}^{(1)}$. We will in fact only need that $l_{1} \leq m+1$. Then the first iterated measure $\sigma_{1}=J\left(\sigma_{0}\right)$ is supported on $\Sigma_{1}$ and has a density $v_{1}$ satisfying

$$
\int_{\Sigma_{1}} \log |x-t| v_{1}(t) d t=Q(x)-F_{1}, \quad x \in \Sigma_{1},
$$

with some constant $F_{1}$. Differentiating this, we get the singular integral equation on several intervals

$$
\int_{\Sigma_{1}} \frac{v_{1}(t)}{x-t} d t=Q^{\prime}(x), \quad x \in \Sigma_{1},
$$

which has the general solution (see [6], p. 427, formula (42.29))

$$
v_{1}(t)=\frac{1}{\pi R_{1}(t)}\left[\pi_{l_{1}-1}(t)+\frac{1}{\pi} \int_{\Sigma_{1}} \frac{Q^{\prime}(s)}{s-t} R_{1}(s) d s\right], \quad t \in \Sigma_{1},
$$

where $R_{1}$ is given by (4.12) with $k=1$ and and $\pi_{l_{1}-1}(t)$ is an arbitrary polynomial of degree $\leq l_{1}-1$. This polynomial is uniquely determined by the condition $\int v_{1}(t) d t=$ 1 , along with the $l_{1}-1$ conditions arising from the fact that the integration constant 
$F_{1}$ in (4.14) is the same on each interval $\left[a_{j}^{(1)}, b_{j}^{(1)}\right]$. From Lemma 4.2 and (4.15) it follows that

$$
P_{1}(t):=\pi R_{1}(t) v_{1}(t)
$$

is a polynomial of degree $m+l_{1}-1$ exactly, and therefore has at most this number of zeros in $\Sigma_{1}$. Recalling that $J(\sigma)^{+} \leq \sigma^{+}$, we have $v_{1} \leq v_{0}$ on $\Sigma_{1}$, and since $v_{0}\left(a_{j}^{(1)}\right)=v_{0}\left(b_{j}^{(1)}\right)=0$ for all $j$, except possibly for $a_{1}^{(1)}$ and $b_{l_{1}}^{(1)}$, we find that

$$
v_{1}\left(a_{j}^{(1)}\right) \leq 0 \quad \text { and } \quad v_{1}\left(b_{j-1}^{(1)}\right) \leq 0, \quad j=2, \ldots, l_{1} .
$$

From this it can be deduced that $v_{1}$ is positive on at most $\left[\left(m+l_{1}-1\right) / 2\right]+1$ subintervals of $\Sigma_{1}$. Thus $\Sigma_{2}$ has the form

$$
\Sigma_{2}=\bigcup_{j=1}^{l_{2}}\left[a_{j}^{(2)}, b_{j}^{(2)}\right]
$$

with $l_{2} \leq\left[\left(m+l_{1}-1\right) / 2\right]+1$ and $a_{1}^{(2)}<b_{1}^{(2)}<\cdots<b_{l_{2}}^{(2)}$. Since $l_{1} \leq m+1$, it then easily follows that also

$$
l_{2} \leq m+1 .
$$

We deduce that we can control our algorithm in the sense that the number of intervals remains bounded by $m+1$. Inductively, it follows that for every $k, \Sigma_{k}$ consists of at most $m+1$ intervals and so has the form given by (4.11). Then with $R_{k}$ as in (4.12) and $P_{k}$ as in (4.13) we get from Lemma 4.2 that $P_{k}$ is a polynomial of degree $m+l_{k}-1 \leq 2 m$, which is non-negative on at most $m+1$ subintervals of $\Sigma_{k}$, and $\Sigma_{k+1}$ consists of at most $m+1$ intervals. This proves parts (a) and (b) of the theorem.

Now taking the limit as $k \rightarrow \infty$, we have that $\Sigma^{*}$ given by (4.10) also consists of at most $m+1$ intervals, say

$$
\Sigma^{*}=\bigcup_{j=1}^{l^{*}}\left[a_{j}^{*}, b_{j}^{*}\right], \quad l^{*} \leq m+1,
$$

and $a_{1}^{*} \leq b_{1}^{*}<a_{2}^{*} \leq \cdots \leq b_{l}^{*}$. It could happen that some of the intervals in the limit shrink to a single point. We use $\Sigma$ to denote the set $\Sigma^{*}$ minus its set of isolated points, and we write

$$
\Sigma=\bigcup_{j=1}^{l}\left[a_{j}, b_{j}\right], \quad l \leq l^{*} \leq m+1,
$$

with $a_{1}<b_{1}<\cdots<b_{l}$. The boundary of $\Sigma^{*}$ has capacity zero, and therefore, by the remarks preceding Theorem 4.1, the iterated balayage algorithm converges. This proves part (c).

It also follows that the equilibrium measure $\mu$ is supported on $\Sigma$ (it cannot have mass at the isolated points of $\left.\Sigma^{*}\right)$ and has density $v(t)=\lim _{k} v_{k}(t), t \in \Sigma$, given by

$$
v(t):=\frac{1}{\pi R(t)} P(t), \quad t \in \Sigma,
$$


with

$$
R(t):=(-1)^{l-j_{0}}\left(\prod_{j=1}^{l}\left|\left(b_{j}-t\right)\left(t-a_{j}\right)\right|\right)^{1 / 2}, \quad t \in\left(a_{j_{0}}, b_{j_{0}}\right),
$$

and $P(t)$ a polynomial of degree $m+l-1$. Hence $v$ does not vanish identically on any subinterval of $\Sigma$, which means that $\Sigma$ is the support of $\mu$. It follows that the support consists of at most $m+1$ intervals. This proves parts (d) and (e), and the proof of the theorem is complete.

We will use the following result in the proof of Theorem 1.1 in the next section.

Theorem 4.3. Let $Q$ be a polynomial external field on $[-1,1]$ and let $\mu$ be the equilibrium measure in the presence of $Q$ with support

$$
\Sigma=\bigcup_{j=1}^{l}\left[a_{j}, b_{j}\right]
$$

(see Theorem 4.1). Let $v$ be the density of $\mu$ on $\Sigma$. Suppose $\Sigma \subset[a, b]$ for some interval $[a, b] \subset[-1,1]$, and let $v_{0}$ be defined as in (4.4).

(a) Suppose that there exist a number $b^{*} \in[a, b)$ such that $\left[a, b^{*}\right] \subset \operatorname{supp}(\mu)$ and $a t_{0} \in\left(b^{*}, b\right)$ such that $\sqrt{(b-t)(t-a)} v_{0}(t)$ decreases on $\left[b^{*}, t_{0}\right]$. Then

(1) $\operatorname{supp}(\mu) \cap\left[a, t_{0}\right]=\left[a_{1}, b_{1}\right]$ is an interval containing $a$, and

(2) $\sqrt{(b-t)(t-a)} v(t)$ decreases on $\left[b^{*}, b_{1}\right]$.

(b) Suppose that there exist a number $a^{*} \in(a, b]$ such that $\left[a^{*}, b\right] \subset \operatorname{supp}(\mu)$ and $a t_{0} \in\left(a, a^{*}\right)$ such that $\sqrt{(b-t)(t-a)} v_{0}(t)$ increases on $\left[t_{0}, a^{*}\right]$. Then

(1) $\operatorname{supp}(\mu) \cap\left[t_{0}, b\right]=\left[a_{l}, b_{l}\right]$ is an interval containing $b$, and

(2) $\sqrt{(b-t)(t-a)} v(t)$ increases on $\left[a_{l}, a^{*}\right]$.

Proof. It is enough to prove part (a), since part (b) follows from (a) by symmetry. So assume the conditions of part (a) are satisfied. Let $d \sigma_{0}(t):=v_{0}(t) d t$ and let $\Sigma_{k}$ and $\sigma_{k}, k=1,2, \ldots$, be given by (4.7). We also put $\Sigma_{0}=[a, b]$. We use $v_{k}$ to denote the density of $\sigma_{k}$ on $\Sigma_{k}$, which exists by Theorem 4.1 (b).

From Theorem 4.1 (a) we know that each $\Sigma_{k}$ consists of a finite number of intervals, which we denote as in (4.11). Since $\operatorname{supp}(\mu) \subset \Sigma_{k}$ for each $k$, we have

$$
\left[a, b^{*}\right] \subset \Sigma_{k}, \quad k=1,2, \ldots,
$$

and it follows that for the endpoints of the first interval $\left[a_{1}^{(k)}, b_{1}^{(k)}\right]$ of $\Sigma_{k}$, we have

$$
a_{1}^{(k)}=a, \quad b_{1}^{(k)} \geq b^{*} .
$$

Using mathematical induction, we show that for each $k=0,1, \ldots$,

(I) $\Sigma_{k} \cap\left[a, t_{0}\right]=\left[a, b_{1}^{(k)}\right]$, where $b_{1}^{(0)}=t_{0}$;

(II) $\sqrt{(b-t)(t-a)} v_{k}(t)$ decreases on $\left[b^{*}, b_{1}^{(k)}\right]$.

For $k=0$ this follows immediately from the assumptions of the theorem.

Let $k \geq 0$ and assume (I) and (II) hold for $k$. Using $\Sigma_{k+1}=\operatorname{supp}\left(\sigma_{k}^{+}\right)$and (II), we find that $\Sigma_{k+1} \cap\left[b^{*}, b_{1}^{(k)}\right]$ is an interval. In view of (4.16) with $k$ replaced by $k+1$, we then have

$$
\Sigma_{k+1} \cap\left[b^{*}, b_{1}^{(k)}\right]=\left[b^{*}, b_{1}^{(k+1)}\right] .
$$


From (I) and the fact that $\Sigma_{k+1} \subset \Sigma_{k}$, it then follows that

$$
\Sigma_{k+1} \cap\left[a, t_{0}\right]=\Sigma_{k+1} \cap\left[a, b_{1}^{(k)}\right]=\left[a, b_{1}^{(k+1)}\right],
$$

which is (I) with $k+1$ instead of $k$.

Next, we note that

$$
\sigma_{k+1}=J\left(\sigma_{k}\right)=\sigma_{k}^{+}-\operatorname{Bal}\left(\sigma_{k}^{-} ; \Sigma_{k+1}\right)
$$

and therefore

$$
v_{k+1}(t)=v_{k}(t)-u_{k}(t), \quad t \in \Sigma_{k+1},
$$

where $u_{k}$ is the density of $\operatorname{Bal}\left(\sigma_{k}^{-} ; \Sigma_{k+1}\right)$. From Theorem 3.1, we get that

$$
\sqrt{\left(b_{l_{k}}^{(k)}-t\right)(t-a)} u_{k}(t)
$$

increases on $\left[a, b_{1}^{(k+1)}\right]$, and so a fortiori on the smaller interval $\left[b^{*}, b_{1}^{(k+1)}\right]$. Here $b_{l_{k}}^{(k)}$ is the largest number in $\Sigma_{k}$. Since $b_{l_{k}}^{(k)} \leq b$, we easily get that

$$
\sqrt{(b-t)(t-a)} u_{k}(t)
$$

increases on $\left[b^{*}, b_{1}^{(k+1)}\right]$. Combining this with (II) and (4.17), we obtain (II) with $k$ replaced by $k+1$.

Thus (I) and (II) hold for every $k$. Then we get from Theorem 4.1 (d) and (I) that $\operatorname{supp}(\mu) \cap\left[a, t_{0}\right]$ is a single interval, which proves (1). From (II) and the fact that $v(t)=\lim _{k} v_{k}(t)$ we find (2). This completes the proof of part (a).

\section{Proof of Theorem 1.1}

In this section, we prove Theorem 1.1. We need two lemmas. The first is the classical Descartes' rule of signs.

Lemma 5.1. The number of zeros on $(0, \infty)$ of any polynomial

$$
\sum_{i=0}^{n} a_{i} t^{i}
$$

is at most the number of sign changes in the sequence of coefficients $\left(a_{i}\right)_{i=0}^{n}$ after zero terms are discarded.

Proof. See for example [1] or [10].

The next lemma is of a computational nature.

Lemma 5.2. Let $m \geq 1$.

(a) For every $a \in[-1,1)$, we have

$$
\frac{1}{\pi} \int_{a}^{1} \frac{s^{2 m}}{s-t} \sqrt{(1-s)(s-a)} d s=\sum_{j=0}^{2 m} B_{j}(a) t^{j}-t^{2 m+1}, \quad a<t<1,
$$

with coefficients $B_{j}(a)$ satisfying

$$
B_{j}(a) \geq 0, \quad j=0,1, \ldots, 2 m, \quad a \in[-1,1) .
$$

Equality holds in (5.2) if and only if $a=-1$ and $j$ is even. 
(b) We have

$$
\frac{1}{\pi} \int_{-1}^{1} s^{2 m} \sqrt{\frac{1-s}{1+s}} d s=\frac{\left(\frac{1}{2}\right)_{m}}{m !}
$$

where $(x)_{m}$ is the Pochhammer symbol given by $(x)_{m}=\Gamma(x+m) / \Gamma(x)$.

Proof. From Lemma 4.2 we know that the left-hand side of (5.1) is a polynomial of degree $2 m+1$ exactly, say with coefficients $B_{j}(a), j=0, \ldots, 2 m+1$. We write

$$
\begin{aligned}
\frac{1}{\pi} \int_{a}^{1} \frac{s^{2 m}}{s-t} \sqrt{(1-s)(s-a)} d s= & \frac{1}{\pi} \int_{a}^{1} \frac{s^{2 m}-t^{2 m}}{s-t} \sqrt{(1-s)(s-a)} d s \\
& +\frac{1}{\pi} \int_{a}^{1} \frac{t^{2 m}}{s-t} \sqrt{(1-s)(s-a)} d s .
\end{aligned}
$$

For the first integral on the right-hand side of (5.4), we find that

$$
\frac{1}{\pi} \int_{a}^{1} \frac{s^{2 m}-t^{2 m}}{s-t} \sqrt{(1-s)(s-a)} d s=\sum_{j=0}^{2 m-1} t^{j} \frac{1}{\pi} \int_{a}^{1} s^{2 m-1-j} \sqrt{(1-s)(s-a)} d s,
$$

and for the second integral, formula 3.228 .3 of [8] gives

$$
\frac{1}{\pi} \int_{a}^{1} \frac{t^{2 m}}{s-t} \sqrt{(1-s)(s-a)} d s=t^{2 m}\left(\frac{1+a}{2}-t\right), \quad a<t<1 .
$$

Thus $B_{2 m+1}(a)=-1, B_{2 m}(a)=(1+a) / 2 \geq 0$ and

$$
B_{j}(a)=\frac{1}{\pi} \int_{a}^{1} s^{2 m-1-j} \sqrt{(1-s)(s-a)} d s, \quad j=0,1, \ldots, 2 m-1 .
$$

It is clear that $B_{j}(a)>0$ if $j$ is odd or if $a \geq 0$, since then the integrand in (5.5) is non-negative. So assume $j$ is even, and $\leq 2 m-2$ and $a<0$. Then we split the integral for $B_{j}(a)$ in (5.5) into an integral from 0 to 1 and an integral from $a$ to 0 . In the second integral we make the change of variables $s \mapsto a s$, and we obtain

$$
\begin{aligned}
B_{j}(a)= & \frac{1}{\pi} \int_{0}^{1} s^{2 m-1-j} \sqrt{(1-s)(s-a)} d s \\
& -(-a)^{2 m+\frac{1}{2}-j} \frac{1}{\pi} \int_{0}^{1} s^{2 m-1-j} \sqrt{(1-s)(1-a s)} d s \\
= & \frac{1}{\pi} \int_{0}^{1} s^{2 m-1-j} \sqrt{1-s}\left(\sqrt{s-a}-(-a)^{2 m+\frac{1}{2}-j} \sqrt{1-a s}\right) d s .
\end{aligned}
$$

Since

$$
\sqrt{s-a} \geq(-a)^{2 m+\frac{1}{2}-j} \sqrt{1-a s}, \quad 0<s<1, \quad-1 \leq a \leq 0,
$$

we see that $B_{j}(a)$ is non-negative for even $j$ and $a \in[-1,0)$. Equality holds in (5.6) only if $a=-1$, which means that equality holds in (5.2) if and only if $j$ is even and $a=-1$. This proves part (a).

For part (b), we note that the change of variables $s \mapsto-s$ gives

$$
\int_{-1}^{1} s^{2 m} \sqrt{\frac{1-s}{1+s}} d s=\int_{-1}^{1} s^{2 m} \sqrt{\frac{1+s}{1-s}} d s
$$


and therefore

$$
\begin{aligned}
\frac{1}{\pi} \int_{-1}^{1} s^{2 m} \sqrt{\frac{1-s}{1+s}} d s & =\frac{1}{2 \pi} \int_{-1}^{1} s^{2 m}\left(\sqrt{\frac{1-s}{1+s}}+\sqrt{\frac{1+s}{1-s}}\right) d s \\
& =\frac{1}{\pi} \int_{-1}^{1} s^{2 m} \frac{1}{\sqrt{1-s^{2}}} d s .
\end{aligned}
$$

The last integral is easily reduced to a beta-integral, and (5.3) follows.

We are ready for:

Proof of Theorem 1.1. We fix $m \in \mathbf{N}, m \geq 1$.

Proof of part (a). Let $c>0$ and $Q_{c}(x)=-c x^{2 m+1}$. We first solve the integral equation (4.1)-(4.2) on the full interval $[-1,1]$. This gives, using (4.4) and (5.1), the representation

$$
\begin{aligned}
v_{0}(t) & =\frac{1}{\pi \sqrt{1-t^{2}}}\left[1-\frac{c(2 m+1)}{\pi} f_{-1}^{1} \frac{s^{2 m}}{s-t} \sqrt{1-s^{2}} d s\right] \\
& =\frac{1}{\pi \sqrt{1-t^{2}}}\left[1-c(2 m+1) \sum_{j=0}^{2 m} B_{j}(-1) t^{j}+c(2 m+1) t^{2 m+1}\right] .
\end{aligned}
$$

All coefficients $B_{j}(-1)$ are non-negative by Lemma 5.2 (a). Thus the polynomial

$$
P_{0}(t):=\pi \sqrt{1-t^{2}} v_{0}(t)
$$

has at most two sign changes in its coefficients. Descartes' rule of signs, see Lemma 5.1 , then gives that $P_{0}$ has at most two positive zeros. Applying Descartes' rule of signs to $P_{0}^{\prime}$, we see that $P_{0}$ has at most one extreme point on $[0, \infty)$. From (5.6), (5.7) we easily get that $P_{0}(0)=1, P_{0}^{\prime}(0)<0$ and

$$
P_{0}(1)=1+\frac{c(2 m+1)}{\pi} \int_{-1}^{1} s^{2 m} \sqrt{\frac{1+s}{1-s}} d s>1 .
$$

It follows that $P_{0}$ has a local minimum at a unique $t_{0} \in(0,1)$, and $P_{0}$ decreases on $\left[0, t_{0}\right]$ and increases on $\left[t_{0}, 1\right]$. We see that $P_{0}$ has its maximum on $[-1,1]$ at +1 and, by symmetry, since $P_{0}(t)-1$ is an odd function (which is immediate from (5.7), (5.8)), its minimum at -1 . Lemma $5.2(\mathrm{~b})$ yields that

$$
P_{0}(-1)=1-\frac{c(2 m+1)}{\pi} \int_{-1}^{1} s^{2 m} \sqrt{\frac{1-s}{1+s}} d s=1-c(2 m+1) \frac{\left(\frac{1}{2}\right)_{m}}{m !} .
$$

Thus $P_{0}$ is non-negative on $[-1,1]$, and $S_{c}=[-1,1]$ if and only if

$$
c \leq c_{1}(m):=\frac{m !}{(2 m+1)\left(\frac{1}{2}\right)_{m}} .
$$

This proves part (a).

In the rest of the proof we consider for various combinations of $a \in(-1,1)$ and $c>0$ the integral equation (4.1), (4.2) on $[a, 1]$ with $Q(x)=Q_{c}(x)$. This leads by (4.4) to a function $v_{0}$ depending on the parameters $a$ and $c$, given by

$$
v_{0}(t ; a, c)=\frac{1}{\pi \sqrt{(1-t)(t-a)}}\left[1-\frac{c(2 m+1)}{\pi} \int_{a}^{1} \frac{s^{2 m}}{s-t} \sqrt{(1-s)(s-a)} d s\right] .
$$


The associated polynomial $P_{0}(t ; a, c)$, defined by

$$
P_{0}(t ; a, c):=\pi \sqrt{(1-t)(t-a)} v_{0}(t ; a, c),
$$

has by Lemma 5.2 (a) the expansion

$$
P_{0}(t ; a, c)=1-c(2 m+1) \sum_{j=0}^{2 m} B_{j}(a) t^{j}+c(2 m+1) t^{2 m+1}
$$

with $B_{j}(a)>0$. Applying Descartes' rule of signs to $P_{0}^{\prime}$, see Lemma 5.1, we see that $P_{0}(t ; a, c)$ has at most one extreme point in $(0, \infty)$. We note that by $(5.12)$

$$
P_{0}(0 ; a, c)=1-c(2 m+1) B_{0}(a)<1, \quad P_{0}^{\prime}(0 ; a, c)=-c(2 m+1) B_{1}(a)<0,
$$

and by (5.10) and (5.11),

$$
P_{0}(1 ; a, c)=1+\frac{c(2 m+1)}{\pi} \int_{a}^{1} s^{2 m} \sqrt{\frac{s-a}{1-s}} d s>1 .
$$

Therefore, there is a unique $t_{0}$ in $(0,1)$ such that

$$
\left\{\begin{array}{lll}
P_{0}(t ; a, c) & \text { decreases on } & {\left[0, t_{0}\right]} \\
P_{0}(t ; a, c) & \text { increases on } & {\left[t_{0}, 1\right] .}
\end{array}\right.
$$

It is easy to see that $t_{0}$ depends only on $a$ and not on $c$. Therefore we will also write $t_{0}=t_{0}(a)$.

Proof of part (b). Let $c \geq c_{1}(m)$. We solve the integral equation (4.1)-(4.2) on $[a, 1]$ with $-1 \leq a \leq 0$. This gives $v_{0}(t ; a, c)$ on $[a, 1]$ as in (5.10). To obtain a relationship between $c$ and $a$, we impose the condition $v_{0}(a ; a, c)=0$, from which we get that $c=C(a)$ with

$$
C(a):=\left(\frac{2 m+1}{\pi} \int_{a}^{1} s^{2 m} \sqrt{\frac{1-s}{s-a}} d s\right)^{-1} .
$$

The polynomial $P_{0}(t ; a, C(a))$, see (5.11), then depends analytically on $a$. For $a=-1$, we have from (5.9) and (5.14) that $C(a)=c_{1}(m)$, and it follows from the proof of part (a) that $P_{0}(t ;-1, C(-1))>0$ for $t \in(-1,1]$. By continuity we also have, for $a$ slightly bigger than -1 , that $P_{0}(t ; a, C(a))>0$ for $t \in(a, 1]$. Define

$$
\mathcal{A}:=\left\{a_{0} \in[-1,0]: \text { for all } a \in\left[-1, a_{0}\right], P_{0}(t ; a, C(a)) \geq 0 \text { on }[a, 1]\right\},
$$

which is clearly a closed interval $\mathcal{A}=\left[-1, a^{*}\right]$ with

$$
a^{*}:=\max \mathcal{A} \in(-1,0] \text {. }
$$

We show that for $a \in \mathcal{A}$ and $c=C(a)$, we have $S_{c}=[a, 1]$. Indeed, for such $a$ and $c$, the measure $d \mu(t):=v_{0}(t ; a, c) d t$ is a probability measure on $[a, 1]$ (cf. (4.2)) and by $(4.1)$

$$
U^{\mu}(x)+Q_{c}(x)=F_{c}, \quad x \in[a, 1],
$$

for some constant $F_{c}$. On $[-1, a)$ we have

$$
\frac{d^{2}}{d x^{2}} U^{\mu}(x)=\int_{a}^{1} \frac{v_{0}(t ; a, c)}{(t-x)^{2}} d t>0, \quad x \in[-1, a) .
$$


Hence $U^{\mu}$ is convex on $[-1, a)$, and $Q_{c}(x)=-c x^{2 m+1}$ is convex on $[-1, a)$ as well, since $a \leq 0$. Next, we have

$$
\frac{d}{d x} U^{\mu}(x)=\int_{a}^{1} \frac{v_{0}(t ; a, c)}{t-x} d t,
$$

which is a singular integral if $x \in(a, 1)$ and an ordinary integral if $x \in[-1, a)$. Since $v_{0}(t ; a, c)=\mathcal{O}\left((t-a)^{1 / 2}\right)$ as $t \searrow a$, we find that $(d / d x) U^{\mu}(x)$ is continuous at $x=a$. Then because of (5.17), we have

$$
\left(U^{\mu}+Q_{c}\right)^{\prime}(a)=0 .
$$

From the convexity of $U^{\mu}+Q_{c}$ on $[-1, a)$, we then get

$$
\left(U^{\mu}+Q_{c}\right)^{\prime}(x)<0, \quad x \in[-1, a) .
$$

It follows that $U^{\mu}+Q_{c}$ is strictly decreasing on $[-1, a)$, and so by $(5.17)$

$$
U^{\mu}(x)+Q_{c}(x)>F_{c} \quad x \in[-1, a) .
$$

The relations (5.17) and (5.19) show that $\mu=\mu_{c}$, the equilibrium measure with external field $Q_{c}$, and thus $S_{c}=[a, 1]$, whenever $a \in \mathcal{A}$ and $c=C(a)$. From the strict inequality in (5.19) we find that also $S_{c}^{*}=[a, 1]$, see $(2.1)$.

From the uniqueness of $S_{c}$, we get that $a \mapsto C(a)$ is strictly increasing for $a \in \mathcal{A}$. Now we put

$$
c_{2}(m):=C\left(a^{*}\right),
$$

and it follows from the above that for every $c \in\left(c_{1}(m), c_{2}(m)\right]$ there is an $a \in$ $\left(-1, a^{*}\right]$ with $c=C(a)$ and

$$
S_{c}=S_{c}^{*}=[a, 1], \quad c \in\left(c_{1}(m), c_{2}(m)\right] .
$$

To complete the proof of part (b), it remains to show that $a^{*}<0$, and for this it suffices to prove that $0 \notin \mathcal{A}$, see (5.16). But, if $0 \in \mathcal{A}$, then we can follow the above arguments, and it would follow that (5.18) holds for $a=0$. Since $Q_{c}^{\prime}(0)=0$ we get $\left(U^{\mu}\right)^{\prime}(0)=0$, which gives a contradiction, since

$$
\frac{d}{d x} U^{\mu}(0)=\int_{0}^{1} \frac{v_{0}(t ; 0, C(0))}{t} d t>0 .
$$

This completes the proof of part (b).

For the remaining parts of the proof, we introduce

$$
a_{1}(c):=\min S_{c}, \quad c>0 .
$$

We already know from part (a) that $a_{1}(c)=-1$ if $c \leq c_{1}(m)$, and from part (b) that $a_{1}(c)$ increases from -1 to $a^{*}$ as $c$ increases from $c_{1}(m)$ to $c_{2}(m)$. Moreover, using Remark 2.1, we see that $a_{1}(c)$ increases and is continuous from the right. We define

$$
c_{3}(m):=\inf \left\{c: a_{1}(c)>0\right\} .
$$

We now proceed to prove part (d), and after that part (c).

Proof of part (d). Let $c>c_{3}(m)$, so that $S_{c} \subset(0,1]$ by (5.21) and (5.22). Then it follows that the equilibrium measure with external field $Q_{c}$ on $[-1,1]$ is the same as the equilibrium measure with external field $Q_{c}$ on $[0,1]$. This was considered in [11], see also Remark 1.4 (1), and it follows that $S_{c}$ consists of at most two intervals. If it were two intervals, then one interval would contain 0 ; but this is not possible, 
since $S_{c} \subset(0,1]$. Thus $S_{c}$ consists of exactly one interval, and has the form $\left[a_{1}, 1\right]$ with $a_{1}=a_{1}(c) \in(0,1)$.

For $c=c_{3}(m)$, we then get by continuity from the right, see Remark 2.1, that $S_{c_{3}}=\left[a^{* *}, 1\right]$ with

$$
a^{* *}:=a_{1}\left(c_{3}(m)\right) \geq 0 .
$$

It is not possible that $a^{* *}=0$, since then we find a contradiction in the same way that $a^{*}=0$ gave a contradiction in the proof of part (b). Thus $a^{* *}>0$, and this completes the proof of part (d).

Proof of part (c). We define

$$
\gamma:=\gamma(m):=\sup \left\{c>0: 0 \in S_{c}\right\} .
$$

From (5.20) and the proof of part (b), we have that $S_{c_{2}}=\left[a^{*}, 1\right]$ with $a^{*}<0$. Likewise, from the proof of part (d), we have $S_{c_{3}}=\left[a^{* *}, 1\right]$ with $a^{* *}>0$. Thus $c_{2} \leq \gamma \leq c_{3}$. We first prove that we have strict inequalities here.

To show that $c_{2}<\gamma$, we use the polynomial $P_{0}\left(t ; a^{*}, c_{2}\right)$ given by $(5.10)-(5.11)$ with $a^{*}$ defined in (5.16). Since $a^{*} \in \mathcal{A}$ and $c_{2}=C\left(a^{*}\right)$, we have that $P_{0}\left(t ; a^{*}, c_{2}\right) \geq$ 0 for $t \in\left[a^{*}, 1\right]$, see (5.15) and (5.20). From (5.13) we obtain that $P_{0}\left(t ; a^{*}, c_{2}\right)$ does not have its minimum at $t=0$, and therefore

$$
P_{0}\left(0 ; a^{*}, c_{2}\right)>0 \text {. }
$$

It follows that the density of the equilibrium measure $\mu_{c_{2}}$ (which is equal to $v_{0}\left(t ; a^{*}, c_{2}\right)$, see $\left.(5.10)\right)$ is strictly positive at $t=0$. Then Lemma 2.3 implies that $0 \in S_{d}$ for $d>c_{2}$ sufficiently close to $c_{2}$. Hence $\gamma>c_{2}$.

To prove $\gamma<c_{3}$, we note first that it follows from the proof of part (d) that $S_{c_{3}}=\left[a^{* *}, 1\right]$, and therefore

$$
\begin{cases}U^{\mu_{c_{3}}}(x)+Q_{c_{3}}(x)=F_{c_{3}}, & x \in\left[a^{* *}, 1\right] \\ U^{\mu_{c_{3}}}(x)+Q_{c_{3}}(x) \geq F_{c_{3}}, & x \in[-1,1] .\end{cases}
$$

If equality held for $x=0$, i.e., if $0 \in S_{c_{3}}^{*}$, then we would get

$$
\left(U^{\mu_{c_{3}}}+Q_{c_{3}}\right)^{\prime}(0)=0
$$

and this would give a contradiction, since

$$
\frac{d}{d x} U^{\mu_{c_{3}}}(0)=\int_{a^{* *}}^{1} \frac{1}{t} d \mu_{c_{3}}(t)>0
$$

and $Q_{c_{3}}^{\prime}(0)=0$. Thus $0 \notin S_{c_{3}}^{*}$, and then it follows from (2.5) that $0 \notin S_{d}$ for $d<c_{3}$ sufficiently close to $c_{3}$. This proves $\gamma<c_{3}$, and so we have

$$
c_{2}(m)<\gamma(m)<c_{3}(m) \text {. }
$$

We continue now with the case $c \in\left[c_{2}, \gamma\right)$. Since $S_{c} \subset\left[a_{1}(c), 1\right]$, we may start to solve the integral equation (4.1)-(4.2) on $\left[a_{1}, 1\right]$ with $a_{1}=a_{1}(c)$. We get $v_{0}\left(t ; a_{1}, c\right)$ as given by (5.10) and the polynomial $P_{0}\left(t ; a_{1}, c\right)$ as in (5.11). We obtain from (5.24) and $c<\gamma$ that $0 \in S_{c}$. From the convexity of the external field $Q_{c}(x)=-c x^{2 m+1}$ on $[-1,0]$, it follows that $S_{c} \cap[-1,0]$ is an interval, see Theorem IV.1.10 of [18]. Hence

$$
S_{c} \cap[-1,0]=\left[a_{1}(c), 0\right], \quad c_{2} \leq c<\gamma .
$$


From (5.26) and Lemma 2.3 it follows that the density of $\mu_{c_{2}}$ is strictly positive on $\left[a_{1}(c), 0\right]$ for every $c>c_{2}$. Letting $c \searrow c_{2}$, we find that

$$
P_{0}\left(t ; a^{*}, c_{2}\right)>0, \quad \text { for } t \in\left(a^{*}, 0\right] .
$$

(Recall that $v_{0}\left(t ; a^{*}, c_{2}\right)$ is the density of $\mu_{c_{2}}$.) On the interval $[0,1]$, the polynomial $P_{0}\left(t ; a^{*}, c_{2}\right)$ assumes its minimum at $t_{0}\left(a^{*}\right) \in(0,1)$, see (5.13). Then it follows easily from the fact that $a^{*}=\max \mathcal{A}<0$ that

$$
P_{0}\left(t_{0}\left(a^{*}\right) ; a^{*}, c_{2}\right)=0 .
$$

Using Lemma 2.3, we find that $t_{0}\left(a^{*}\right) \notin S_{c}$ for all $c>c_{2}$. Therefore $P_{0}\left(t ; a_{1}(c), c\right)$ cannot be non-negative on the full interval $\left[a_{1}(c), 1\right]$ if $c \in\left(c_{2}, \gamma\right)$. We find, using (5.13) and (5.26), that $P_{0}\left(t_{0}\left(a_{1}\right) ; a_{1}, c\right)<0$, and so $t_{0}\left(a_{1}\right) \notin S_{c}$. Furthermore, as $P_{0}\left(t ; a_{1}, c\right)$ decreases on $\left[0, t_{0}\left(a_{1}\right)\right]$, we get from Theorem 4.3 (a) and $(5.26)$ that $S_{c} \cap\left[a_{1}, t_{0}\left(a_{1}\right)\right]$ is an interval. Thus there is a $b_{1}=b_{1}(c) \in\left[0, t_{0}\left(a_{1}\right)\right)$ such that

$$
S_{c} \cap\left[-1, t_{0}\right]=\left[a_{1}, b_{1}\right] .
$$

We also have, using $(5.13)$, that $P_{0}\left(t ; a_{1}, c\right)$ increases on $\left[t_{0}\left(a_{1}\right), 1\right]$. Then by Theorem 4.3 (b) we get, since $1 \in S_{c}$,

$$
S_{c} \cap\left[t_{0}, 1\right]=\left[a_{2}, 1\right]
$$

for some $a_{2}=a_{2}(c) \in\left(t_{0}, 1\right]$. Combining (5.27) and (5.28), we see that

$$
S_{c}=\left[a_{1}(c), b_{1}(c)\right] \cup\left[a_{2}(c), 1\right],
$$

and we have proved part (c) for $c \in\left(c_{2}, \gamma\right)$.

Next, we have $\gamma<c_{3}$ by (5.25), and thus $a_{1}(\gamma)<0$. Then, letting $c$ increase towards $\gamma$, we obtain from (5.29) that

$$
S_{\gamma}=\left[a_{1}(\gamma), b_{1}(\gamma)\right] \cup\left[a_{2}(\gamma), 1\right] .
$$

If $b_{1}(\gamma)>0$, then it would follow from Theorem 4.3 (a) (2) that

$$
\pi \sqrt{(1-t)\left(t-a_{1}\right)} v(t),
$$

with $v$ the density of the equilibrium measure $\mu_{\gamma}$, decreases on $\left[0, b_{1}(\gamma)\right]$ and $v(0)>$ 0 . Then Lemma 2.3 implies that $0 \in S_{d}$ for some $d>\gamma$. This is impossible by (5.24), and therefore $b_{1}(\gamma)=0$. Thus we get

$$
S_{\gamma}=\left[a_{1}(\gamma), 0\right] \cup\left[a_{2}(\gamma), 1\right] .
$$

Now to the remaining case $c \in\left(\gamma, c_{3}\right)$. Here we solve the integral equation (4.1)(4.2) on $[a, 1]$ with $a=a_{1}(\gamma)$. This gives us the polynomial $P_{0}\left(t ; a_{1}(\gamma), c\right)$ which depends on $c$, but its minimum on $[0,1]$ is at the same point $t_{0}=t_{0}\left(a_{1}(\gamma)\right)$, see (5.13). On $\left[t_{0}, 1\right], P_{0}\left(t ; a_{1}(\gamma), c\right)$ is increasing, and therefore by Theorem $4.3(\mathrm{~b})$, $S_{c} \cap\left[t_{0}, 1\right]$ is an interval containing 1 . Hence

$$
S_{c} \cap\left[t_{0}, 1\right]=\left[a_{2}(c), 1\right] .
$$


Since $S_{c} \subset S_{\gamma}$, we find that $a_{2}(c)>a_{2}(\gamma)$. Then we also get, because of (5.30),

$$
S_{c} \cap(0,1]=\left[a_{2}(c), 1\right] .
$$

Because the external field $Q_{c}$ is convex on $[-1,0]$, we have that $S_{c} \cap[-1,0]$ is an interval, see Theorem IV.1.10 of [18]. It is non-empty since $\min S_{c}<0$. Hence

$$
S_{c} \cap[-1,0]=\left[a_{1}(c), b_{1}(c)\right]
$$

with $-1<a_{1}(c)<b_{1}(c)<0$. (Observe that $0 \notin S_{c}$.) From (5.31), (5.32), we have

$$
S_{c}=\left[a_{1}(c), b_{1}(c)\right] \cup\left[a_{2}(c), 1\right]
$$

and this proves part (c) for the remaining case $c \in\left(\gamma, c_{3}\right)$.

Now the proof of Theorem 1.1 is complete, and we are done.

\section{REFERENCES}

1. P. Borwein and T. Erdélyi, Polynomials and Polynomial Inequalities, Springer-Verlag, New York, 1995. MR 97e:41001

2. S. B. Damelin and D. S. Lubinsky, Jackson theorems for Erdös weights in $L_{p}(0<p \leq \infty)$, J. Approx. Theory 94 (1998), 333-382. CMP 98:17

3. P. Deift, T. Kriecherbauer and K. T-R McLaughlin, New results on the equilibrium measure for logarithmic potentials in the presence of an external field, J. Approx. Theory 95 (1998), 388-475. CMP 99:05

4. P. Deift and K. T-R McLaughlin, A continuum limit of the Toda lattice, Mem. Amer. Math. Soc. 624 (1998). MR 98h:58076

5. Z. Ditzian and D. S. Lubinsky, Jackson and smoothness theorems for Freud weights in $L_{p}$ $(0<p \leq \infty)$, Constr. Approx. 13 (1997), 99-152. MR 97k:41028

6. F.D. Gakhov, Boundary Value Problems, Pergamon Press, Oxford, 1966. MR 33:6311

7. A. A. Gonchar and E. A. Rakhmanov, Equilibrium measure and the distribution of zeros of extremal polynomials, Mat. Sb. 125 (1984), 117-127. English transl: Math. USSR-Sb. 53 (1986), 119-130. MR 86f:41002

8. I. S. Gradshteyn and I. M. Ryzhik, Table of Integrals, Series, and Products, Academic Press, Orlando, 1980. MR 81g:33001

9. K. G. Ivanov and V. Totik, Fast decreasing polynomials, Constr. Approx. 6 (1990), 1-20. MR 90k:26023

10. S. Karlin and W. J. Studden, Tchebycheff Systems with Applications in Analysis and Statistics, Wiley, New York, 1966. MR 34:4757

11. A. B. J. Kuijlaars and P. D. Dragnev, Equilibrium problems associated with fast decreasing polynomials, Proc. Amer. Math. Soc. 127 (1999), 1065-1074. CMP 99:06

12. A. B. J. Kuijlaars and W. Van Assche, A problem of Totik on fast decreasing polynomials, Constr. Approx. 14 (1998), 97-112. MR 99b:26026

13. G. G. Lorentz, M. von Golitschek and Y. Makovoz, Constructive Approximation, Advanced Problems, Springer-Verlag, Berlin, 1996. MR 97k:41002

14. D. S. Lubinsky and E. B. Saff, Strong Asymptotics for Extremal Polynomials associated with Weights on $\mathbb{R}$, Lect. Notes in Math. vol. 1305, Springer-Verlag, Berlin, 1988. MR 89m:41013

15. H. N. Mhaskar, Introduction to the Theory of Weighted Polynomial Approximation, World Scientific, Singapore, 1996. MR 98i:41014

16. H. N. Mhaskar and E. B. Saff, Where does the sup norm of a weighted polynomial live?, Constr. Approx. 1 (1985), 71-91. MR 86a:41004

17. E. A. Rakhmanov, On asymptotic properties of polynomials orthogonal on the real axis, Mat. Sb. 119 (1982), 163-203. English transl.: Math. USSR-Sb. 47 (1984), 155-193. MR 84e:42025

18. E. B. Saff and V. Totik, Logarithmic Potentials with External Fields, Springer-Verlag, New York, 1997. CMP 98:05

19. H. Stahl and V. Totik, General Orthogonal Polynomials, Cambridge University Press, Cambridge, 1992. MR 93d:42029 
20. V. Totik, Weighted Approximation with Varying Weight, Lect. Notes in Math. vol. 1569, Springer-Verlag, Berlin, 1994. MR 96f: 41002

21. V. Totik, Fast decreasing polynomials via potentials, J. Anal. Math. 62 (1994), 131-154. MR 96e: 41010

Department of Mathematics, University of the Witwatersrand, PO Wits 2050, South AFRICA

E-mail address: 036sbd@cosmos.wits.ac.za

Department of Mathematics, Katholieke Universiteit Leuven, Celestijnenlaan 200 B, B-3001 Leuven, Belgium

E-mail address: arno@wis.kuleuven.ac.be 